\title{
The Remodeling of Synaptic Extracellular Matrix and Its Dynamic Relationship with Nerve Terminals at Living Frog Neuromuscular Junctions
}

\author{
Lanlin Chen, Debra Brown Folsom, and Chien-Ping Ko \\ Department of Biological Sciences, Section of Neurobiology, University of Southern California, Los Angeles, \\ California 90089-2520
}

The question of whether the synaptic extracellular matrix undergoes remodeling and how this remodeling is related to nerve terminal plasticity was examined in living neuromuscular junctions of adult frogs. Sartorius muscles were double stained with a fluorescent nerve terminal dye 4-(4diethylamino-styryl)- $\boldsymbol{N}$-methylpyridinium iodide (4-Di-2-Asp) and rhodamine-tagged peanut agglutinin (PNA) which recognizes synaptic extracellular matrix. Both nerve terminals and synaptic extracellular matrix in $\mathbf{2 0 0}$ identified normal junctions were visualized in vivo two or three times over a period of 2.6-6 months. The majority of neuromuscular junctions (NMJs) showed remodeling of both nerve terminals and synaptic extracellular matrix. Only $2.5 \%$ showed no changes in either synaptic element. The most commonly seen remodeling involved correlated changes in both nerve terminals and synaptic extracellular matrix. In this large group, while some junctions $(20 \%)$ showed overall proportionate changes in all branches, most junctions (68\%) showed disproportionate extension and/or retraction of some but not all individual branches. Another group of NMJs $(9.5 \%)$ showed mismatched changes in the nerve terminal and synaptic extracellular matrix. In this group, some NMJs showed a decrease in the nerve terminal length without a corresponding reduction in synaptic extracellular matrix length. In other junctions that displayed extension of branches, the PNA-stained matrix was longer than the distal tip of the nerve terminal. Morphometric analysis indicated an average increase of $15.6 \%$ in total nerve terminal length and $13.6 \%$ in total synaptic extracellular matrix length. Although almost all NMJs displayed remodeling in at least one branch, about $\mathbf{5 0} \%$ of the $\mathbf{2 2 0 1}$ individual branches examined did not show changes. The average change was $8.9 \%$ growth in the length of individual nerve terminal branches and $8.3 \%$ growth in the length of individual branches of synaptic extracellular matrix. There was no significant difference in the morphometry between the repeatedly observed junctions and the previously unobserved control junctions. Furthermore, junctions in which the synaptic extracellular matrix was longer than

Received Feb. 22, 1991 ; revised Apr. 22, 1991; accepted Apr. 24, 1991.

This work was supported by NIH Grant NS 17954. We thank R. Balice-Gordon, A. Herrera, L. Holcomb, and J. Lichtman for critical comments on the manuscript. Correspondence should be addressed to Dr. Chien-Ping Ko, Department of Biological Sciences, Section of Neurobiology, Hedco Neuroscience Building, University of Southern California, Los Angeles, CA 90089-2520.

Copyright @ 1991 Society for Neuroscience $0270-6474 / 91 / 112920-11 \$ 03.00 / 0$ the nerve terminal also were seen in control as well as in experimental muscles. Cases where the nerve terminals were longer than the synaptic extracellular matrix were never observed in newly arising junctional branches. The present study has shown extensive remodeling in not only the nerve terminal but also the synaptic extracellular matrix in adult living frog NMJs. Results suggest that nerve terminals retract before the synaptic extracellular matrix. A hypothesis that extension of synaptic extracellular matrix precedes nerve terminal growth during synaptic remodeling is proposed.

Synaptic connections in the normal adult nervous system are thought to be undergoing continuous remodeling throughout the animal's lifetime (Grinnell and Herrera, 1981; Purves and Lichtman, 1985; Wernig and Herrera, 1986; Robbins, 1988). Early work using histological staining of fixed muscle tissues (Wernig et al., 1980; Anzil et al., 1984) showed nerve endings unopposed by postsynaptic specializations or vice versa in the frog neuromuscular junction (NMJ). These structures were interpreted as evidence for synaptic sprouting and retraction, respectively, in normal muscles. More direct evidence showing synaptic remodeling in normal adult tissues has been obtained only recently by repeated observations of the same synapses over a period of time in living animals. This direct approach has become possible due to several newly developed techniques, including vital dyes for the nerve terminal (Yoshikami and Okun, 1984; Magrassi et al., 1987), low-light-level video microscopy, and digital image processing (Inoue, 1986). Using these techniques, Purves and colleagues have shown substantial changes in the morphology of pre- or postsynaptic elements in the autonomic ganglia of mature mice (Purves et al., 1986, 1987; Purves and Voyvodic, 1987). However, the arrangement of motor nerve terminals in mouse fast-twitch muscles was quite stable, apart from overall proportionate growth, over periods of several months (Lichtman et al., 1987; Balice-Gordon and Lichtman, 1990). Small changes involving filopodia and additions and deletions of branches of NMJs have been observed in adult mouse slow-twitch muscles (Robbins and Polak, 1988; Wigston, 1989, 1990). In contrast to mouse NMJs, extensive remodeling of nerve terminals including deletion and addition of entire new nerve terminal branches has been shown in adult living frog NMJs (Herrera and Werle, 1990; Herrera et al., 1990). These results were similar to those suggested by earlier histological and ultrastructural studies in frog muscles (Wernig et al., 1980; Anzil et al., 1984). The extensive remodeling seen in adult frog NMJs provides an opportunity to examine further the cel- 
lular and molecular bases of synaptic plasticity, especially the relationship between nerve terminals and the extracellular matrix (ECM) in the synapse.

The ECM has been shown to play important roles in various aspects of development and differentiation in the nervous system (Sanes, 1989; Jessell et al., 1990). At reinnervated NMJs, the ECM has been shown to direct the differentiation of preand postsynaptic specializations (Sanes et al., 1978; Burden et al., 1979; reviewed by Sanes, 1989). An ECM molecule, agrin, has been shown to play a role in aggregation of $\mathrm{ACh}$ receptors at NMJs (Nitkin et al., 1987). Recently, another ECM molecule called s-laminin has been identified at mammalian NMJs and shown to be inhibitory to neurite outgrowth (Hunter et al., 1989; Weis et al., 1989). Thus, remodeling of the ECM in the NMJ may also play a profound role in the maintenance and plasticity of motor nerve terminals. However, whether the synaptic ECM undergoes remodeling and how the synaptic ECM is related to the nerve terminal during synaptic remodeling in normal adult animals have not been examined.

Prior to investigating these questions, it was essential to find a specific probe for the synaptic ECM. Since the ECM is rich in carbohydrates, lectins have been used to recognize NMJs (Sanes and Cheney, 1982; Kelly et al., 1985; Ko, 1987; Scott et al., 1988). In frog skeletal muscles, we have found that peanut agglutinin (PNA), which recognizes D-galactose- $\beta-(1-3) N$-acetylgalactosamine (Lotan et al., 1975), is the most specific lectin probe for NMJs (Ko, 1987, 1991). Electron microscopic studies using HRP-labeled PNA (HRP-PNA) have revcalcd that PNAbinding molecules (PNA-BMs) are located in the ECM in the synaptic cleft and junctional folds and around Schwann cells at the NMJ (Ko, 1987; Ko and Folsom, 1990; Somasekhar and Ko, 1991). In addition, fluorescently labeled PNA does not affect synaptic transmission and may be used as a vital probe for living frog NMJs (Ko, 1987). Furthermore, fluorescent PNA can be combined with vital fluorescent dyes for the nerve terminal such as 3,3' diethyloxadicarbocyanine iodide $\left(\mathrm{DiOC}_{2}\right)$ and 4-(4-diethylamino-styryl)- $N$-methylpyridium iodide (4-Di-2-Asp) (Yoshikami and Okun, 1984; Magrassi et al., 1987) to visualize independently the synaptic ECM and nerve terminal in the same NMJ (Ko, 1987; Chen and Ko, 1988). Thus, double staining with fluorescent PNA and nerve terminal dye provides a unique approach to examine not only the remodeling of the synaptic ECM itself but also the dynamic relationship between the synaptic ECM and the nerve terminal in NMJs of living animals.

In the present study, identified NMJs double stained with 4-Di-2-Asp and rhodamine-conjugated PNA were visualized two or three times over $2.6-6$ months. The results reported here indicate that the synaptic ECM at frog NMJs undergoes continuous remodeling during the growth and retraction of nerve terminals. Although most NMJs show correlated changes between these two synaptic elements, instances where the synaptic ECM is longer than the nerve terminal are seen at some NMJs undergoing retraction as well as at junctions undergoing extension.

Preliminary reports of these results have been published (Chen and Ko, 1988; Ko, 1991).

\section{Materials and Methods}

Experiments were performed on adult frogs (Rana pipiens) kept in the laboratory. For viewing NMJs, frogs $(22-40 \mathrm{gm})$ were anesthetized by immersing in $0.2 \%$ tricaine methanesulfonate (Sigma) for $20-30 \mathrm{~min}$ and then cooled with ice. An incision was made in one leg to expose the sartorius muscle, which was rinsed frequently with frog Ringer's solution (111 mM NaCl, $2 \mathrm{~mm} \mathrm{KCl}, 1.8 \mathrm{~mm} \mathrm{CaCl}_{2}, 5 \mathrm{~mm}$ HEPES, $\mathrm{pH}$ 7.2). A strip of Kimwipe tissue soaked with frog Ringer's solution containing $10 \mu \mathrm{M}$ of the fluorescent dye 4-(4-diethylamino-styryl)- $N$-methylpyridinium iodide (4-Di-2-Asp; Molecular Probes) was applied to the muscle for 3 min (Magrassi et al., 1987; Herrera and Banner, 1990). This was followed by staining with tetramethylrhodamine isothiocyanate-labeled peanut agglutinin (PNA; $50 \mu \mathrm{g} / \mathrm{ml}$ in Ringer's solution) for $30 \mathrm{~min}$ (Ko, 1987). After rinsing the stained muscle with Ringer's solution, the frog was placed on an aluminum plate designed to keep the temperature at $0-1^{\circ} \mathrm{C}$ by connecting the periphery of the plate to a recirculating chiller (Herrera and Banner, 1990). This low temperature reduced the heart beat frequency and movement caused by the heart beat and prolonged the anesthesia of the frog. The cooling plate was attached to the stage of a compound microscope equipped for epifluorescence (Olympus BH2-RFL).

For viewing rhodamine-PNA stained NMIs, the standard green excitation/red emission unit, (BP-545)/(EY-550), supplemented with a barrier filter, (R-610), was used. For 4-Di-2-Asp-stained nerve terminals, the standard blue excitation/green emission, (BP-490)/(EY-475) supplemented with a barrier filter, (G-520), was used. Although 4-Di2-Asp staining "bleeds" through the fluorescence filters for rhodamine the nerve terminals were not apparent when rhodamine-PNA was viewed. This is due to extremely bright fluorescence of rhodamine-PNA staining relative to the weak crossover fluorescence of 4-Di-2-Asp staining. The method to locate NMJs is as follows. The nerve entry (the point where the nerve enters the sartorius muscle) was first located with a $10 \times$ objective and oblique incident illumination from a fiber optics light. The general arrangement of the end-plate region was then quickly scanned for a few seconds using the epifluorescent optics for rhodamine-PNA, which revealed bright staining of NMJs even at this low magnification. The positions of these junctional areas were noted by coordinates on the microscope stage micrometer relative to the position of the nerve entry. Individual NMJs were viewed with a $50 \times$ water-immersion objective lens (Leitz; NA $=1.0$ ) and recorded by a silicon-intensified target (SIT) video camera (Dage SIT 66). To prevent possible damage caused by excessive illumination, a $1.2 \%$ transmittance neutral density filter and a minimum aperture were used, and the exposure time for both PNA and 4-Di-2-Asp was kept to less than a total of $20 \mathrm{sec}$ per NMJ (Magrassi et al., 1987; Herrera and Banner, 1990). The output of the SIT camera was connected to a digital image processor (Image-1, Universal Imaging). Live images were frame averaged and then processed to reduce background and enhance contrast. The processed images were stored on videotape or in digital form on computer diskettes. After 10 $20 \mathrm{NMJs}$ were recorded, the incision was closed with sutures and the frog was returned to its cage. Stored images were photographed from a video monitor.

After 2.6-6 months, frogs were reancsthetized and the muscles werc restained with 4-Di-2-Asp and rhodamine-PNA as described above. NMJs were relocated using the $x-y$ coordinate information, sketch maps of junctional areas, and pictures taken at the initial observations. Most of the identified junctions were viewed twice in vivo; a few were viewed three times. To perform morphometry, nerve terminal staining and PNA staining from initial and final viewings were traced from the monitor screen onto acetate films. Measurements of traced nerve terminals and synaptic ECM were made separately on a digitizing tablet connected to a microcomputer. We measured each branch segment, which is the portion between a distal tip and branch point or between two branch points. We also determined the total junctional length, which is the sum of all individual branches. The percentages of increase or decrease in both nerve terminals and synaptic ECM at subsequent observations of the same junctions were calculated. We define correlation of individual branches as when the difference between the nerve terminal length and the synaptic ECM length of the branch is less than or equal to $3 \mu \mathrm{m}$. When we state that overall changes in both matrix and terminal are correlated over time, we imply that whatever the change in the total synaptic ECM length (increase or decrease), the nerve terminal length changed likewise (or vice versa). Thus, an entire junction may show a correlated increase in size, meaning both the nerve terminal and the synaptic ECM increased in length overall, yet an individual branch of that same NMJ may show lack of correlation, with the synaptic ECM exceeding the length of the nerve terminal by more than $3 \mu \mathrm{m}$.

There were several factors in the experimental procedure that may have influenced NMJ remodeling. In order to exclude the potential effects of 4-Di-2-Asp staining and illumination on morphological changes 

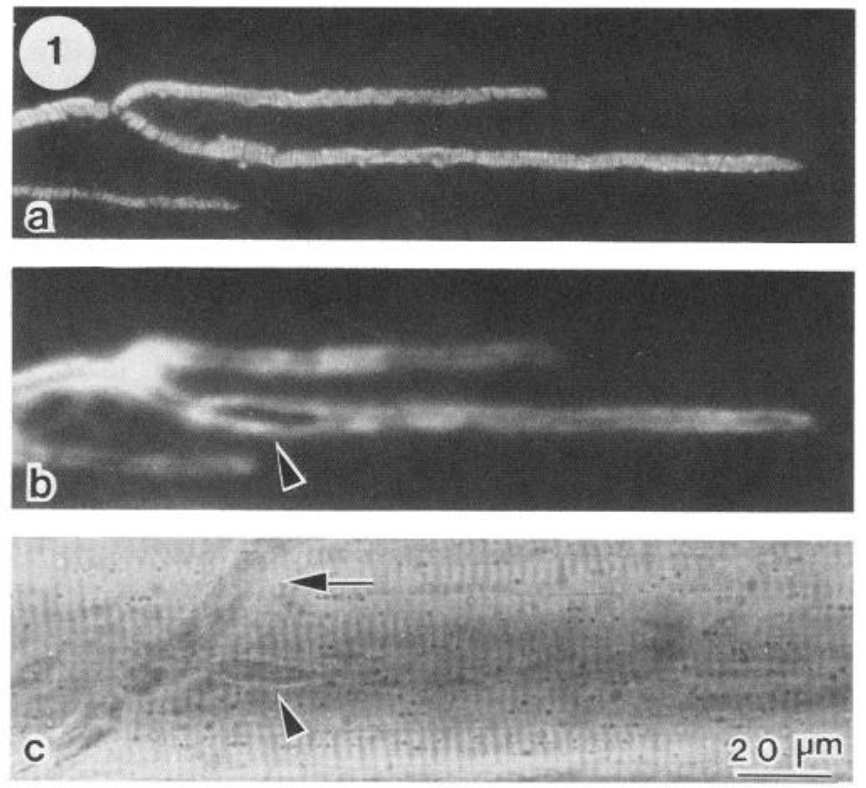

Figure 1. An NMJ was doubly stained with rhodamine- $\alpha$-bungarotoxin $(a)$ and FITC-PNA $(b)$. PNA stains only the NMJ, not the myelinated axon that is indicated by the arrow in $c$. In addition, PNA staining delineates the Schwann cell nucleus (arrowhead in $b$ ) that is seen with bright-field optics of the same region (arrowhead in $c$ ).

of nerve terminals, procedures with precautions to minimize the exposure intensity and duration as used by Herrera et al. (1990) were adopted. In addition, the repeatedly observed junctions in experimental animals were compared with unobserved but repeatedly stained junctions in the same muscle and with junctions in contralateral, previously unobserved muscles to test whether the surgical operation and repeated observations caused remodeling.

\section{Results}

To demonstrate the specific staining of PNA at the NMJ, a frog muscle was double stained with rhodamine- $\alpha$-bungarotoxin (Fig. $1 a$ ) and fluorescein isothiocyanate-PNA (FITC-PNA) (Fig. 1b). As previously shown (Ko, 1987), PNA staining outlined the ACh receptor clusters at the NMJ. PNA did not stain myelinated nerve fibers that could be seen with bright-field optics (arrow in Fig. 1c). PNA staining also delineated the enlarged portion of the Schwann cell body containing the nucleus (arrowheads in Fig. $1 b, c)$. Thus, the migration of Schwann cell nuclei can be revealed with PNA staining.

By repeatedly viewing the same end-plates double stained with 4-Di-2-Asp and rhodamine-PNA over 2.6-6 months, remodeling of both nerve terminals and synaptic ECM was apparent. Several kinds of changes including extension, retraction, de novo formation of branches, and even a combination of these changes were seen within the same NMJ. During the growth and retraction of NMJs, sometimes the nerve terminal and the synaptic ECM both changed correspondingly, and sometimes there was incomplete correlation. Although remodeling was common, not all branches or all NMJs observed showed changes in the nerve terminal and synaptic ECM. In the following sections, junctions are grouped according to the morphological changes they underwent. The terms "synaptic ECM" and "synaptic matrix" used in this report refer to the ECM recognized by PNA.

\section{NMJs showing correlated changes in the nerve terminal and synaptic ECM}

Among 200 repeatedly observed junctions, 176 (90.5\%) showed correlated extension or retraction between the nerve terminal and the synaptic ECM over a period of several months. Some of the simplest changes were uniform expansions $(12.5 \%$ of all junctions) or shrinkages ( $7.5 \%$ of all junctions) of all branches in a junction. As shown in Figure 2, $a$ and $b$, both nerve terminals
N T
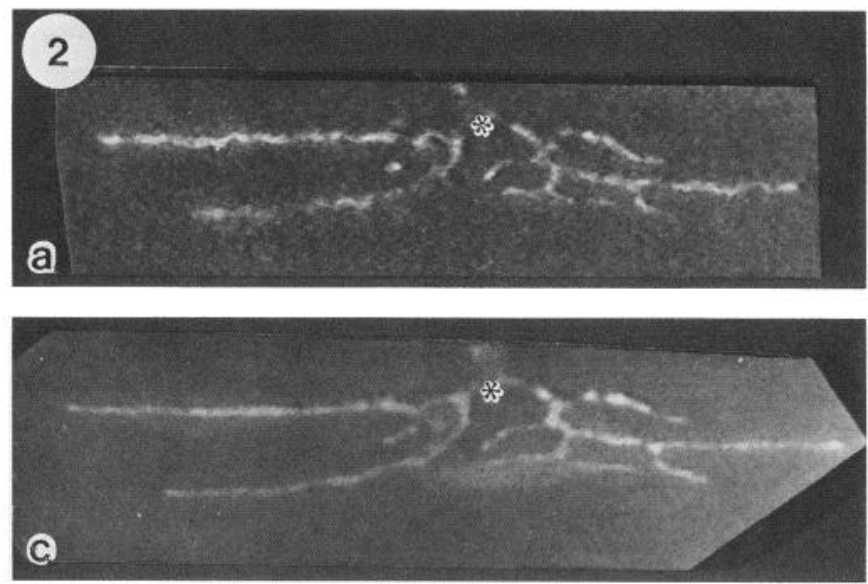

P N A
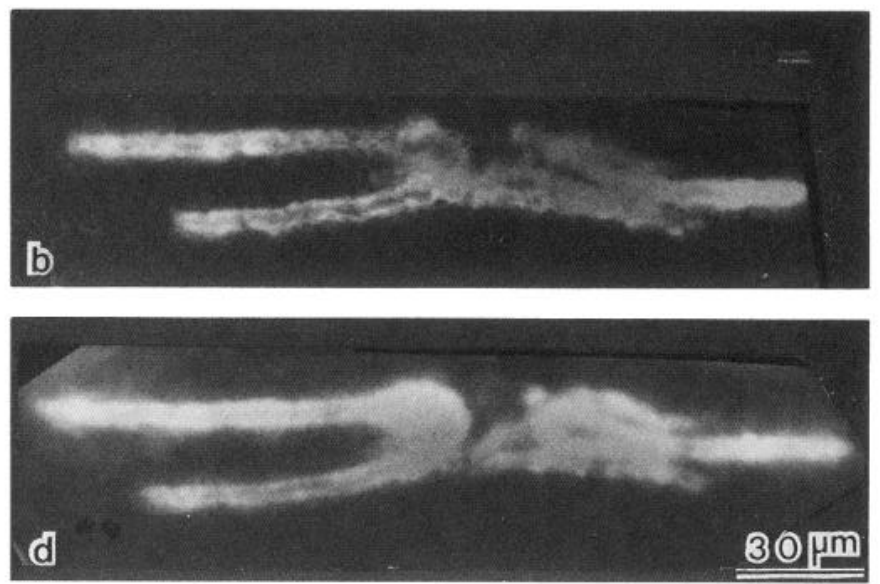

Figure 2. The proportionate enlargement of preexisting branches in both nerve terminal and synaptic matrix. $a$ and $b$, First observation; $c$ and $d$, second observation made $78 \mathrm{~d}$ later. All branches, including the distal tips of the junction and the proximal segments between branch points, are increased in length over time (compare branch lengths in $a$ and $b$ with those in $c$ and $d$, respectively). Asterisks in $a$ and $c$ indicate the end of the myelinated portion of the incoming nerve that was stained with 4-Di-2-Asp but not with PNA. In this and subsequent figures (Figs. 2-7), the left panels (NT) are images of nerve terminals from initial and subsequent viewing(s) in order (from top to bottom), as revealed by 4-Di-2-Asp staining. Right panels $(P N A)$ are images of synaptic ECM from the same fields of double stained muscle in each viewing, as revealed by rhodaminePNA. All images in the same figures have the same magnification. 

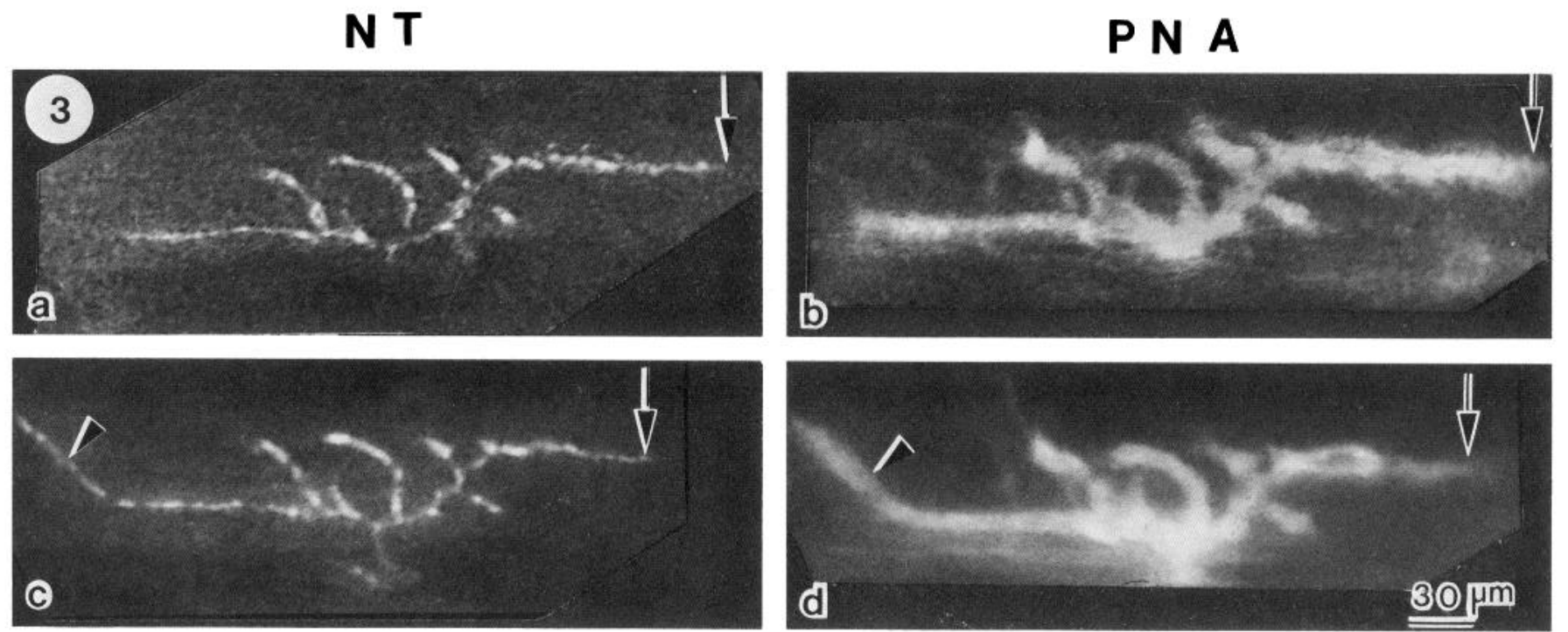

Figure 3. An NMJ with elongation or shortening of some branches, but not others. The initial observations showed nerve terminal (a) matched with synaptic matrix $(b)$. Precise alignment was also seen at the second observation $(c$ and $d) 99 \mathrm{~d}$ later. It was noted that one branch increased in both nerve terminal length (arrowhead in $c$ ) and synaptic matrix length (arrowhead in $d$ ), while another branch decreased in both elements (compare arrows in $a$ and $c$ as well as in $b$ and $d$ ). However, the intercalary branches were not changed over time.

and synaptic matrix matched precisely at the first observation. At the second observation $78 \mathrm{~d}$ later, nerve terminals and synaptic matrix still matched, but both showed proportionate increases in length of preexisting branches without any alteration in the overall branching pattern (Fig. $2 c, d$ ). Such enlargement occurred not only at the ends of branches, but also at most proximal segments between branch points. In Figure 2, the total nerve terminal length and synaptic ECM length were both increased by $14 \%$ at the second observation.

Aside from the uniform changes in the size of the NMJ as described above, the most prevalent changes seen in frog NMJs
(70.5\%) were nonuniform elongation or retraction of individual branches in both nerve terminal and synaptic matrix. Some NMJs had both extension and retraction at different branches in the same junction as shown in Figure 3. At the first observation, nerve terminal staining (Fig. $3 a$ ) was correlated with PNA staining (Fig. $3 b$ ). Over a $99 \mathrm{~d}$ interval, the left branch of the nerve terminal grew out by $38 \mu \mathrm{m}$ along an angle of about 45 degrees relative to the original branch (arrowhead in Fig. $3 c$ ), while the right branch in the same junction retracted $20 \mu \mathrm{m}$ toward the center (arrow in Fig. 3c). In contrast, the medial branches showed little or no changes over time. Meanwhile, the

\section{N T}
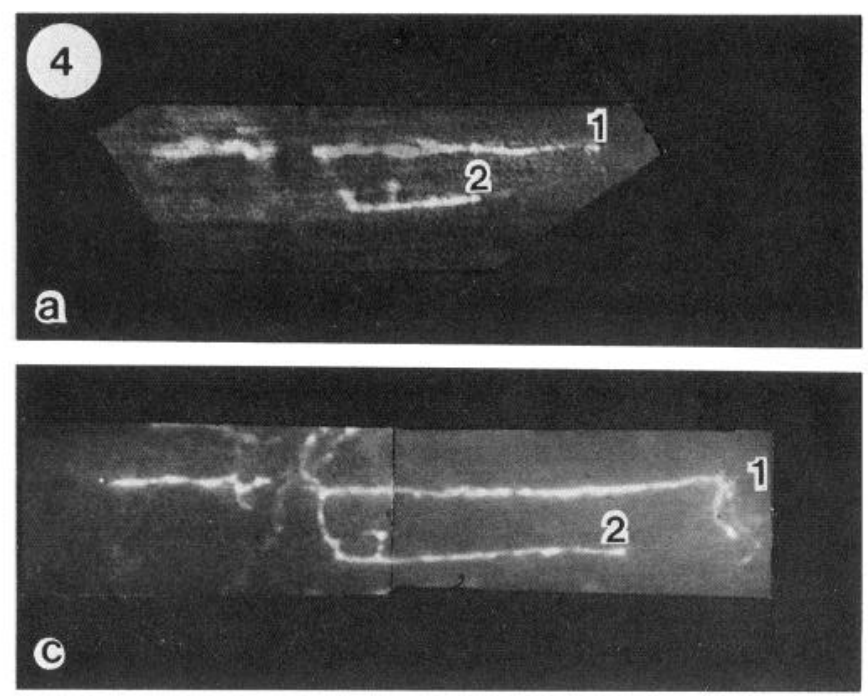

P N A
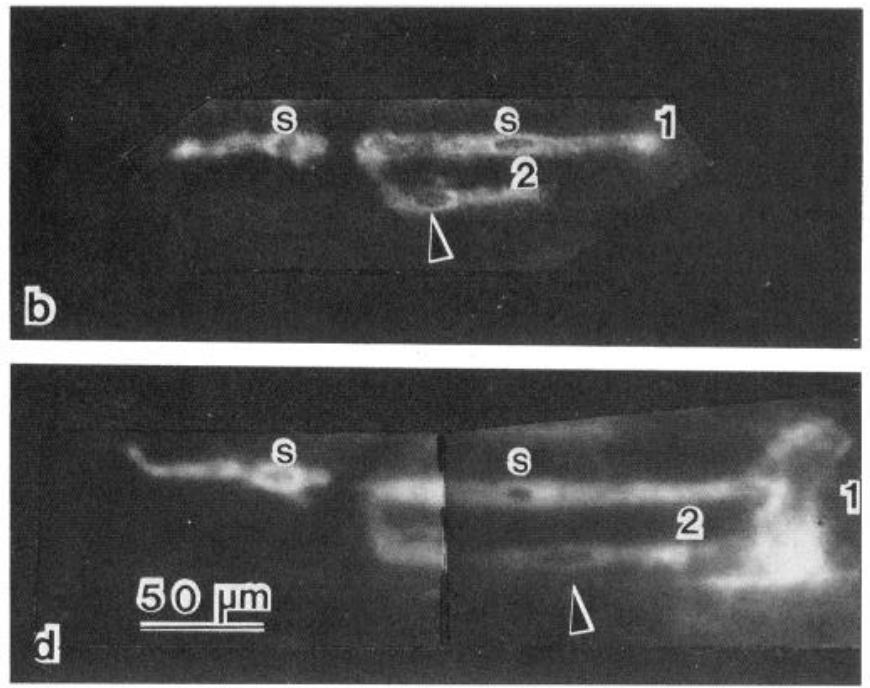

Figure 4. An NMJ underwent an addition of branches over time and showed migration of Schwann cell nuclei. $a$ and $b$, The first viewing of nerve terminal and synaptic matrix, respectively. $s$, Location of Schwann cell nuclei. $c$ and $d$, The second viewing of the same junction, $119 \mathrm{~d}$ later. Note two small terminal branches were formed ( , branch 1); meanwhile, the corresponding synaptic matrix was also expressed, although PNA staining appeared as a large cluster $(d$, branch 1$)$. In addition, the position of one Schwann cell nucleus was changed over time along branch 2 (compare arrowheads in $b$ and $d$ ). 
N T
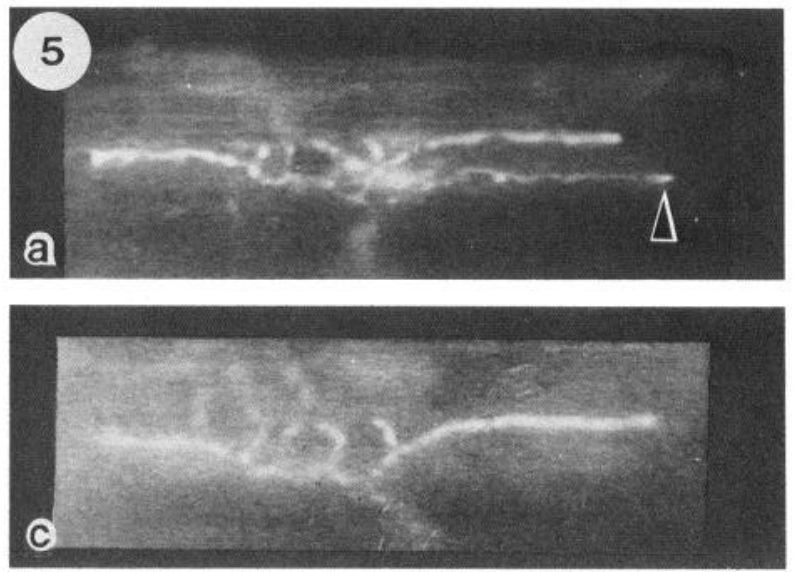

P N A
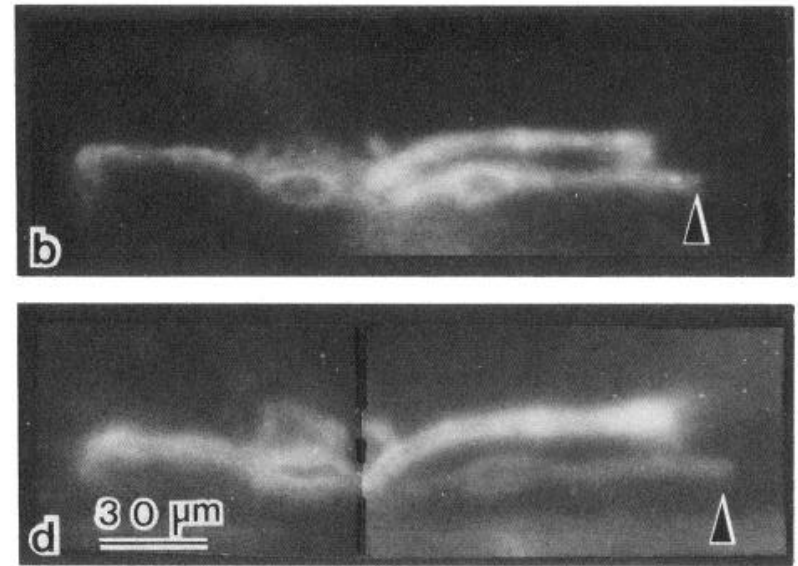

Figure 5. Lack of correlation between nerve terminal and synaptic matrix associated with the retraction of an NMJ. $a$ and $b$, The terminal arbor and corresponding matrix at the initial observation (arrowheads); $c$ and $d$, at the second observation made $184 \mathrm{~d}$ later. One nerve terminal branch indicated by an arrowhead in $a$ was totally deleted; however, the synaptic matrix was still visible at the original site (arrowhead in $d$ ), although the PNA staining became fainter.

synaptic matrix underwent the same kinds of changes as the nerve terminal (arrowhead and arrow in Fig. $3 d$ ) and maintained good correlation with the nerve ending during extension and retraction.

NMJs showing not only extension and even the creation of individual branches, but also a change in the position of an associated Schwann cell body, were seen. As shown in Figure 4 , the initial images displayed good correlation between nerve terminal and synaptic ECM (Fig. $4 a, b$ ), and Schwann cell nuclei were seen ( $\mathrm{s}$ in Fig. $4 b$ ). A second observation made $119 \mathrm{~d}$ later showed the extension of three preexisting branches and the birth of two nerve terminal branches (at the end of branch 1 in Fig. $4 c$ ). Synaptic ECM also appeared at all new junctional sites, but PNA staining was seen as a large, diffuse mass at the tip of branch 1 (Fig. 4d). We often saw such diffuse PNA staining at newly formed junctional areas (also see Fig. $6 d$ ). In Figure 4, a change over time in the location of the Schwann cell nucleus along branch 2 (compare arrowheads in Fig. $4 b, d$ ) was noted, but the nucleus along branch 1 remained in place ( $\mathrm{s}$ in Fig. $4 d$ ).

\section{NMJS showing lack of correlation between nerve terminal and synaptic ECM associated with retraction}

In addition to frequently seen examples of close correspondence of the synaptic ECM with the nerve terminal during growth or retraction of NMJs, we also found some junctions $(9.5 \%$ of all junctions examined) in which the nerve terminal and synaptic ECM did not always change correspondingly. Figure 5 illustrates the disparity between nerve terminal and synaptic ECM during junctional retraction. In the first viewing, PNA staining was correlated with the nerve terminal (Fig. 5a,b). During an interval of $184 \mathrm{~d}$, this junction underwent a small enlargement of both nerve terminal and synaptic matrix that was followed by the deletion of one terminal branch (Fig. $5 c$; refer to the branch indicated by arrowhead in Fig. 5a). PNA staining at the abandoned junctional site is still visible (arrowhead in Fig. $5 d$ ), although the intensity is much fainter than that in the previous viewing (arrowhead in Fig. $5 b$ ). This example suggests that during retraction of the NMJ, the nerve terminal was lost first and the synaptic ECM at the corresponding junctional site was left behind. In the absence of nerve terminal branches, PNA binding was diminished and eventually depleted. The correlation between terminal and synaptic matrix was again attained (see also Fig. 3, arrows).

\section{NMJs showing lack of correlation between nerve terminal and synaptic ECM associated with extension}

Lack of correlation between the nerve terminal and the synaptic ECM was also found during the growth of NMJs. One example is shown in Figure 6. At the initial observation, the synaptic matrix at one branch (arrowhead, Fig. $6 b$ ) was $12 \mu \mathrm{m}$ longer than the nerve terminal (arrowhead, Fig. $6 a$ ). At the second observation made $119 \mathrm{~d}$ later, both nerve terminal and synaptic matrix had extended and even developed new branches (Fig. $6 c, d$, arrowheads). Similar to Figure $4 d$, the PNA staining appeared to be a large diffuse mass around the newly arising terminal branches and more expanded than the terminal branches (compare arrowheads in Fig. $6 c, d$ ). In the final observation (after another $176 \mathrm{~d}$ ), the nerve terminal length stayed the same as in the previous viewing (compare arrowheads in Fig. $6 c, e$ ). The diffuse PNA-stained area now became more strictly confined to the junctional site at one branch (arrowhead in Fig. 6f); however, at another branch, the PNA staining was beyond the distal tip of the nerve terminal branch (Fig. $6 f$, arrow).

Another example of synaptic ECM longer than the nerve terminal at junctional branches undergoing extension is shown in Figure 7. In the initial viewing of this junction, the synaptic ECM colocalized with the nerve terminal (arrowheads in Fig. $7 a, b)$. Similar to the third observation shown in Figure 6 , the second viewing ( $89 \mathrm{~d}$ later) of this junction showed synaptic ECM at one of the branches (arrowhead in Fig. 7d) longer than the nerve terminal, while the nerve terminal length stayed the same as in the first observation (Fig. $7 c$; compare arrowheads in $a$ with $c$ ). However, after another $173 \mathrm{~d}$, this extended synaptic ECM (Fig. $7 f$ ) was now partly occupied by a $30 \mu \mathrm{m}$ bifurcating nerve terminal branch (arrowhead in Fig. 7e). We found that in 323 branches that displayed synaptic ECM longer than the nerve terminal by at least $10 \mu \mathrm{m}$ at the first observation, $63 \%$ showed subsequent extension with the synaptic ECM still 


\section{N T}
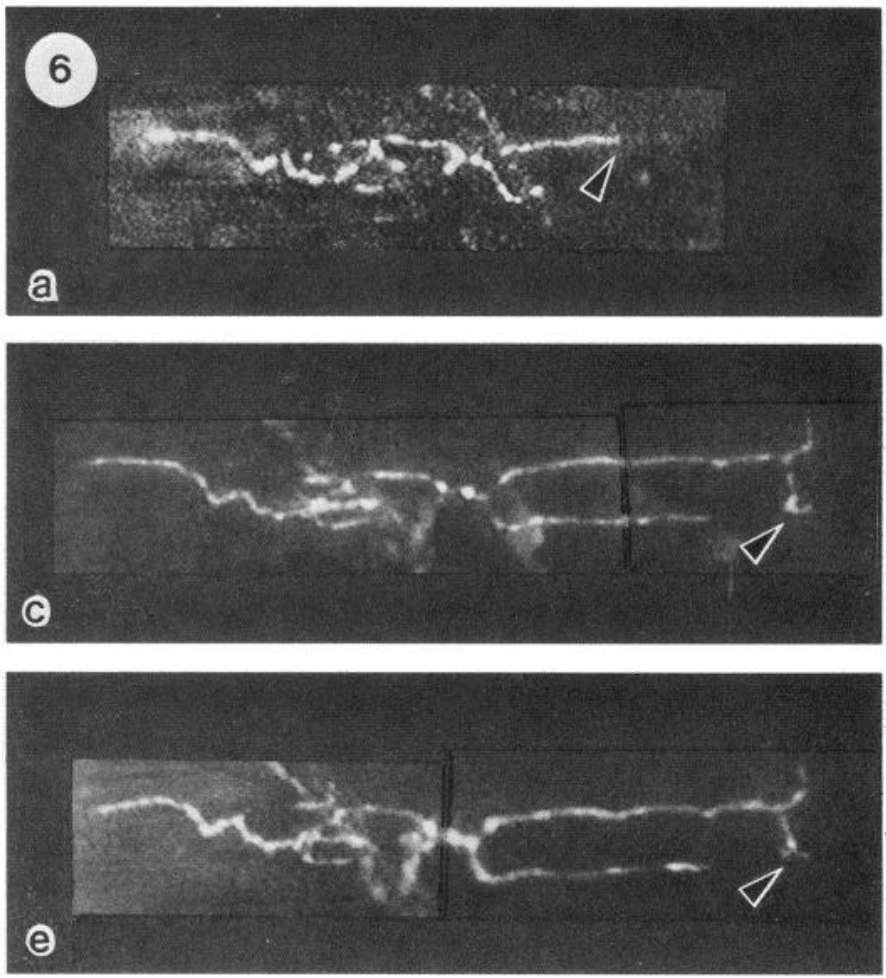

P N A
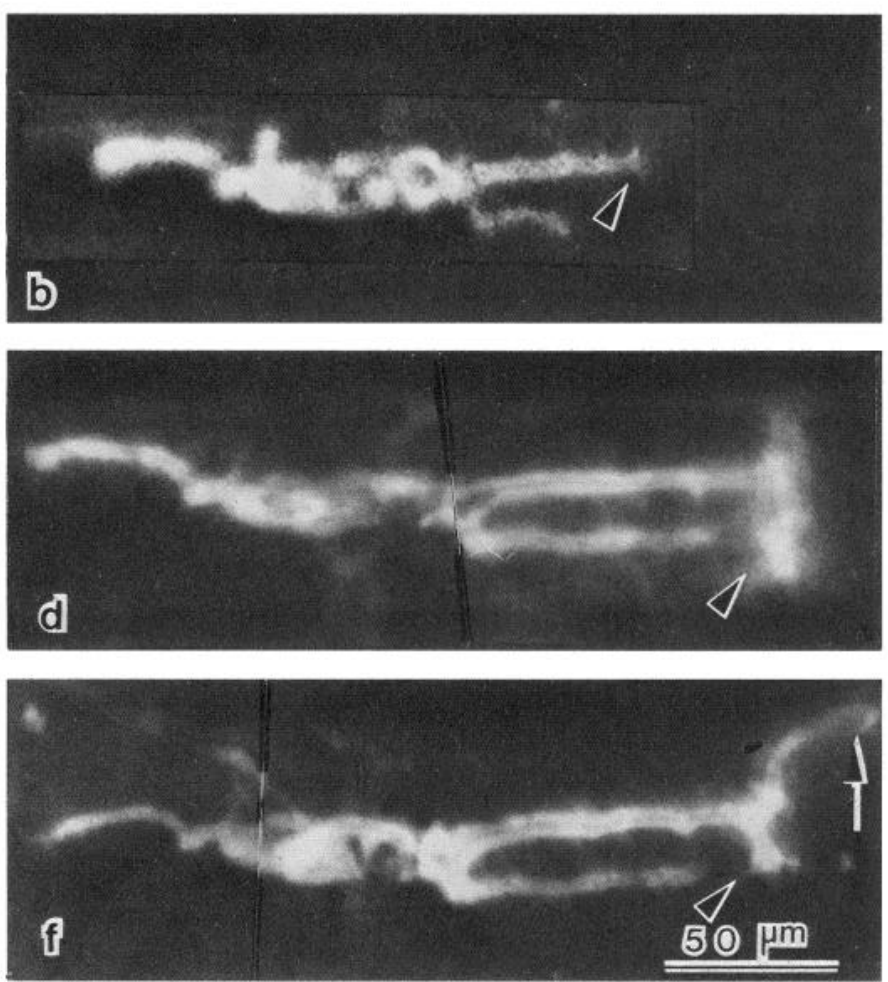

Figure 6. Lack of correlation between nerve terminal and synaptic matrix associated with the growth of NMJ. $a$ and $b$, Original appearance of nerve terminal and synaptic matrix. Arrowheads indicate the tips of a branch undergoing extension. The synaptic matrix was $12 \mu \mathrm{m}$ longer than the nerve terminal at this branch. $c$ and $d$, Same junction, $119 \mathrm{~d}$ later. New terminal branch and corresponding synaptic matrix were seen (arrowheads). $e$ and $f$. Same junction, $176 \mathrm{~d}$ after the previous viewing. Nerve terminal (arrowhead in $e$ ) stayed the same shape and length as in $c$; however, one segment of synaptic matrix (arrow in $f$ ) elongated beyond the nerve terminal while the other segment (arrowhead in $f$ ) was correlated with the nerve terminal.

longer or equal to the terminal at the second observation. No cases were observed where the nerve terminal was longer than the synaptic matrix during the extension of existing branches. One possible interpretation of the result is that synaptic ECM may precede the nerve terminal outgrowth during the extension of junctional branches. However, alternative explanations cannot be ruled out (see Discussion).

\section{NMJs showing lack of correlation between nerve terminal and synaptic ECM in previously unobserved muscles}

The lack of correlation between nerve terminal and synaptic ECM did not occur only in NMJs that were observed repeatedly. It has also been seen in muscles that were not stained nor viewed previously. Figure 8 shows an example of this type of NMJ with 4-Di-2-Asp staining (solid arrowheads in $a$ ) shorter than PNA staining (open arrowheads in $b$ ). Histological staining with the nitroblue tetrazolium method for nerve terminals (Letinsky and DeCino, 1980) and Karnovsky's (1964) cholinesterase technique confirmed the nerve terminal length revealed by 4-Di-2Asp staining and also showed that the nerve terminal (solid arrowheads in Fig. 8c) was shorter than the AChE-labeled region (open arrowheads, $c$ ). The presence of cholinesterase remnants (cholinesterase-stained areas without nerve terminals) shown by the histological staining suggests a retraction of the nerve terminal in this NMJ (Wernig et al., 1980). This result is consistent with the repeated in vivo observations that suggest that the retraction of the nerve terminal occurred before that of the PNAstained matrix.
A lack of correlation between nerve terminal and PNA staining has also been seen in NMJs that might be undergoing extension. Figure 9 shows a branch of an NMJ in which the tip of the PNA-stained matrix (open arrowhead in $b$ ) extended 33 $\mu \mathrm{m}$ beyond the tip of the nerve terminal (solid arrowhead in $a$ ). At the extended, PNA-stained region, neither nerve terminal nor cholinesterase were seen with histological staining (Fig. 9c). In contrast to Figure 8 , the absence of cholinesterase at the extended, PNA-stained region suggests that this region was not previously occupied by the nerve terminal and that the NMJ was not undergoing retraction. Furthermore, there were regions with nerve terminal staining (arrow in Fig. $9 c$ ) but without cholinesterase staining that appeared in other regions (arrowhead) of the junction. This type of NMJ is believed to be associated with sprouting (Wernig et al., 1980). Although direct evidence is lacking, this NMJ could be undergoing growth, and this result would be consistent with the hypothesis that PNA-stained ECM extends in advance of nerve terminal outgrowth.

\section{Morphometry}

We have examined 200 NMJs from 14 frogs for morphological changes in synaptic ECM and nerve terminals; 71 of these junctions were observed three times. Of the NMJs examined between the first and second observations, $72 \%$ showed an increase in the total lengths of nerve terminals and synaptic ECM; $25.5 \%$ showed a decrease in the total length of nerve terminals and synaptic matrix. Only $2.5 \%$ of the NMJs showed no changes in nerve terminal and synaptic ECM. Although changes in the total 


\section{N T}
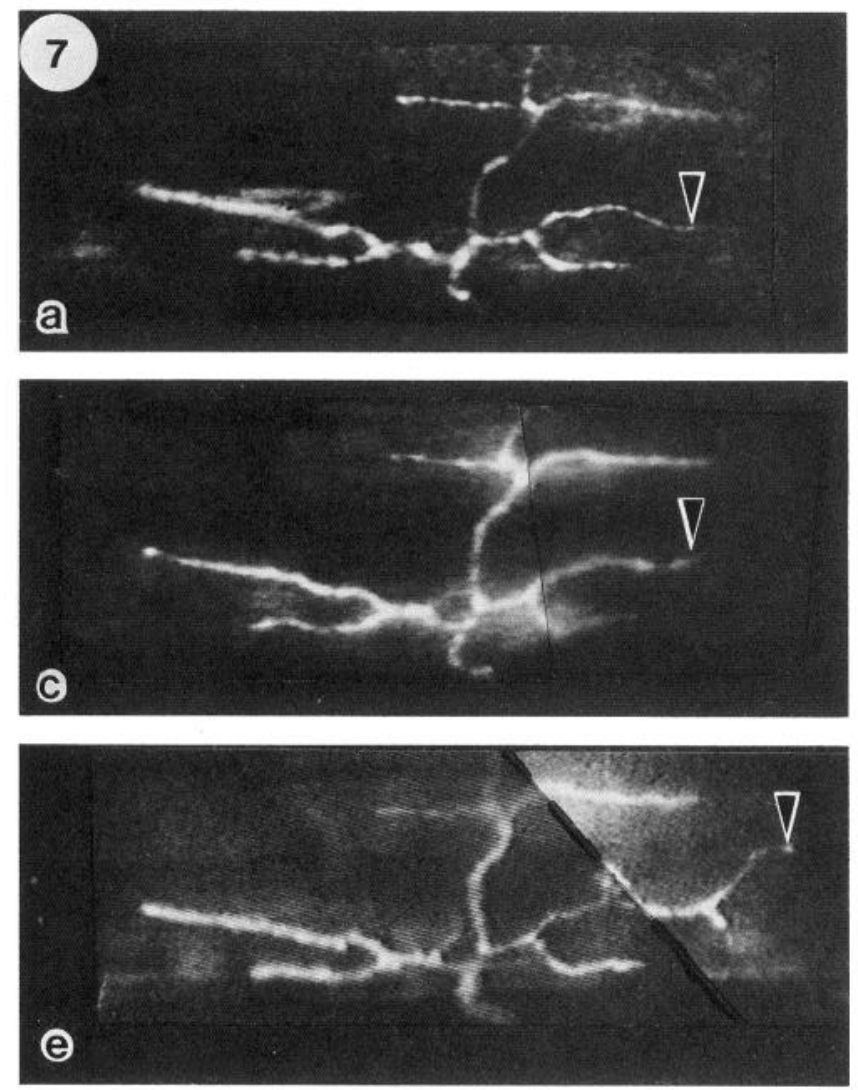

P N A
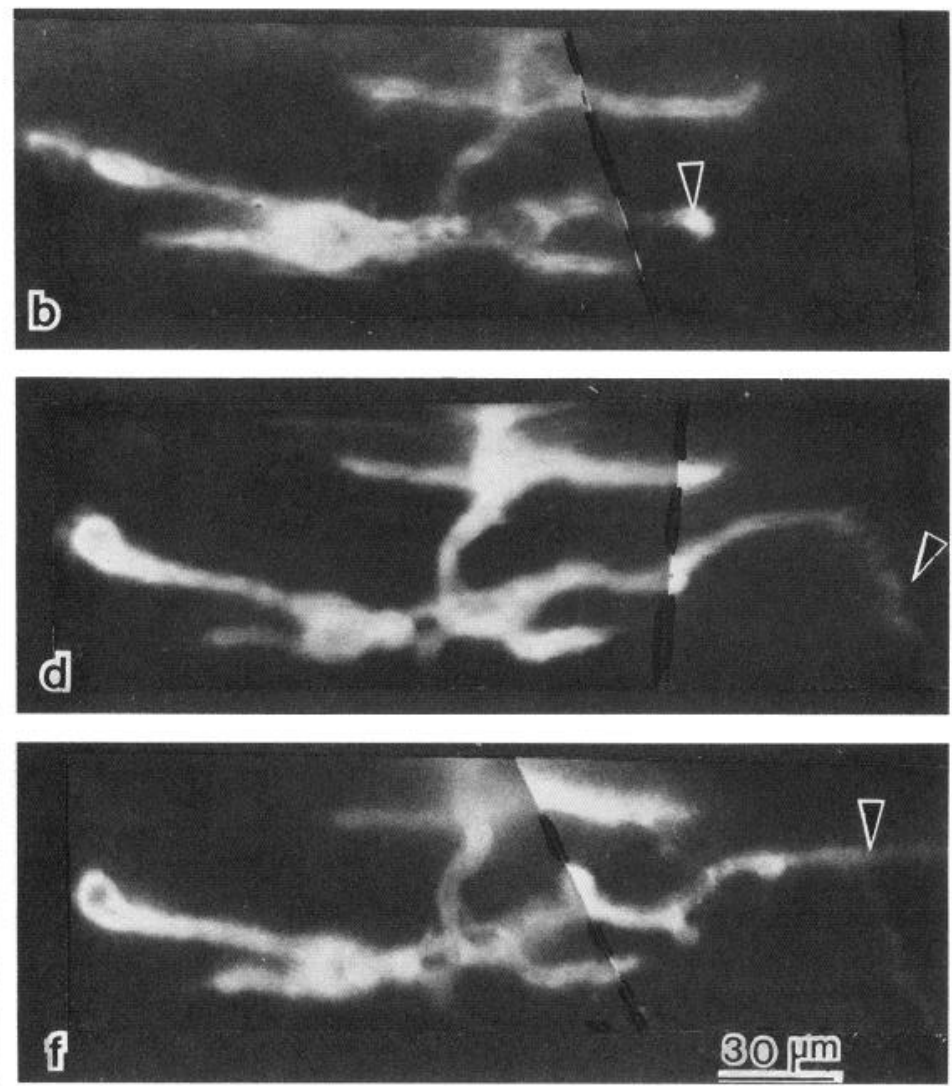

Figure 7. Triple observation of an NMJ showing the growth of a nerve terminal branch following the path of synaptic matrix formed ahead. $a$ and $b$. At the initial viewing, there was close correspondence between nerve terminal and synaptic matrix (arrowheads). $c$ and $d$, The second viewing, $89 \mathrm{~d}$ later. One branch of the synaptic matrix increased in length (arrowhead in $d$ ), while the nerve terminal branch remained unchanged (arrowhead in $c$ ). $e$ and $f$, The final viewing, $173 \mathrm{~d}$ after the second viewing. Note that the nerve terminal (arrowhead in $e$ ) grew into the already formed synaptic matrix region (arrowhead in $f$ ).

junctional sizes were seen in almost all (97.5\%) of the NMJs examined twice, not all of the individual branches of the NMJs showed changes. In these 200 identified NMJs, $50 \%$ of the 2201 branches did not change in nerve terminal branch length, and $46.8 \%$ of synaptic ECM branches also were unchanged between the first and the second observations. Changes of less than or equal to $3 \mu \mathrm{m}$ were considered to be within the margin of error in the measurement procedure so that branches with such changes were considered as remaining unchanged. Of the remainder of the branches, about twice as many showed extension in nerve terminals and synaptic ECM as retraction. The extent of changes in the total lengths of nerve terminals and synaptic ECM was quite variable. This was expressed by the percentage change of increase (positive) or decrease (negative) of the total length of a nerve terminal between first and second observations as shown in a histogram in Figure $10 a$; the percentage changes in total length of synaptic ECM are shown in Figure $10 b$. The mean change in the total nerve terminal length is $15.6 \%$, with a range from $-70 \%$ to $+150 \%$. In the total length of synaptic ECM, the mean percentage change is $13.6 \%$ with a range from $-20 \%$ to $+150 \%$ (Fig. $10 b$ ).

To gain insight into changes in individual branches, each branch was measured and the percentage changes between first and second observations were calculated. Excluding new sprouts and totally disappearing branches (both give $100 \%$ changes re- gardless of the actual magnitude of loss or gain), the distributions of the percentage changes in 1878 preexisting branches of nerve terminal and synaptic matrix in 200 identified NMJs are depicted in Figure 11, $a$ and $b$, respectively. The mean change in the length of individual nerve terminal branches is $8.9 \%$, and the mean change in the length of individual branches of synaptic ECM is $8.3 \%$. About $50 \%$ of branches did not show change in the length of nerve terminal or synaptic ECM during the 2.6-6 month interval between observations. The rest of the branches showed changes ranging from $-60 \%$ to more than $100 \%$ in nerve terminal or synaptic ECM.

In addition to the data shown in Figure 11, newly arising branches were considered. Of 226 branches that arose de novo during the interval between the first and second observations, 74 (33\%) branches showed PNA staining at least $3 \mu \mathrm{m}$ longer than 4-Di-2-Asp staining at the second observation. In all other branches, PNA and 4-Di-2-Asp staining were equal $(<3 \mu \mathrm{m}$ difference). No cases were seen where the nerve terminal exceeded the length of synaptic matrix in newly arising branch. Only 14 junctional branches out of 1878 preexisting branches $(0.7 \%)$ examined had nerve terminal length in excess of synaptic $\mathrm{ECM}$, with a $6 \mu \mathrm{m}$ difference being the greatest.

In order to exclude the possibility that remodeling described in this study is caused by the experimental procedures such as application of fluorescent dyes or epi-illumination, two groups 

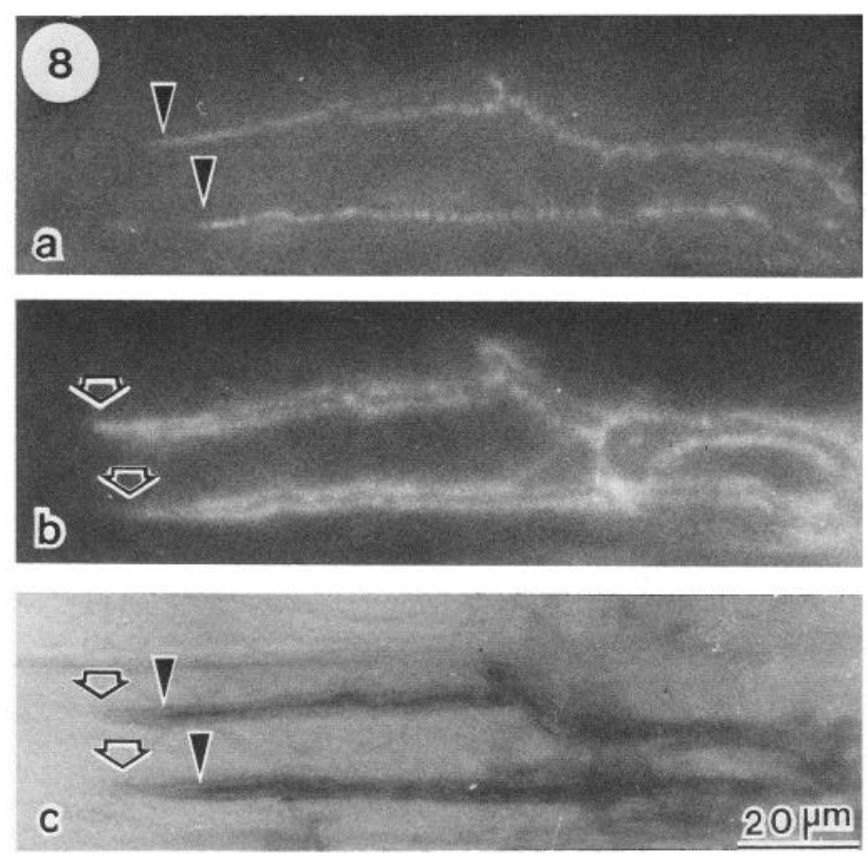

Figure 8. Lack of correlation between nerve terminal and synaptic matrix in an NMJ that was not observed previously. This NMJ was stained with 4-Di-2-Asp $(a)$ and PNA $(b)$, followed by nitroblue tetrazolium staining of the nerve terminal and Karnovsky's cholinesterase staining $(c)$. Lengths of PNA-stained matrix (open arrowheads in $b$ ) at the upper and lower branches are 10 and $13 \mu \mathrm{m}$ longer, respectively, than corresponding nerve terminals (solid arrowheads in $a$ ). These regions were abandoned junctional sites with cholinesterase remnants (open arrowheads in $c$ ) but without nerve terminals. The nitroblue tetrazolium-stained nerve terminals ended at sites shown by solid arrowheads in $c$.

of control experiments were performed. In one group of $10 \mathrm{frogs,}$ as the final observations of the identified NMJs were performed, 161 previously unidentified NMJs located in the same muscles were also examined. The distributions of the total nerve terminal length, the total synaptic ECM length, and the total number of individual branches in the identified NMJs at the final observation were compared with those of the control unidentified NMJs. Results show no significant difference between these two distributions $(p<0.005$, Kolmogorov-Smirnov two-sample test); thus, influence of the illumination procedure on synaptic remodeling is unlikely. In another control, 192 NMJs from 11 contralateral sartorius muscles were examined. These control NMJs were not previously stained, illuminated, or observed. The distributions of the total nerve terminal length, the total synaptic ECM length, and the total number of individual branches between the identified NMJs and the unidentified NMJs in the contralateral muscles are also not significantly different $(p<$ 0.005 , Kolmogorov-Smirnov two-sample test). In addition, branches where the synaptic ECM was longer than the nerve terminal by at least $10 \mu \mathrm{m}$ were seen in $15 \%$ (323 out of 2201) of junctional branches without previous staining and observations. This was similar to $16 \%$ of junctional branches seen at NMJs after repeated observations.

\section{Discussion}

The present study has demonstrated for the first time the remodeling of synaptic ECM in adult living NMJs with repeated,
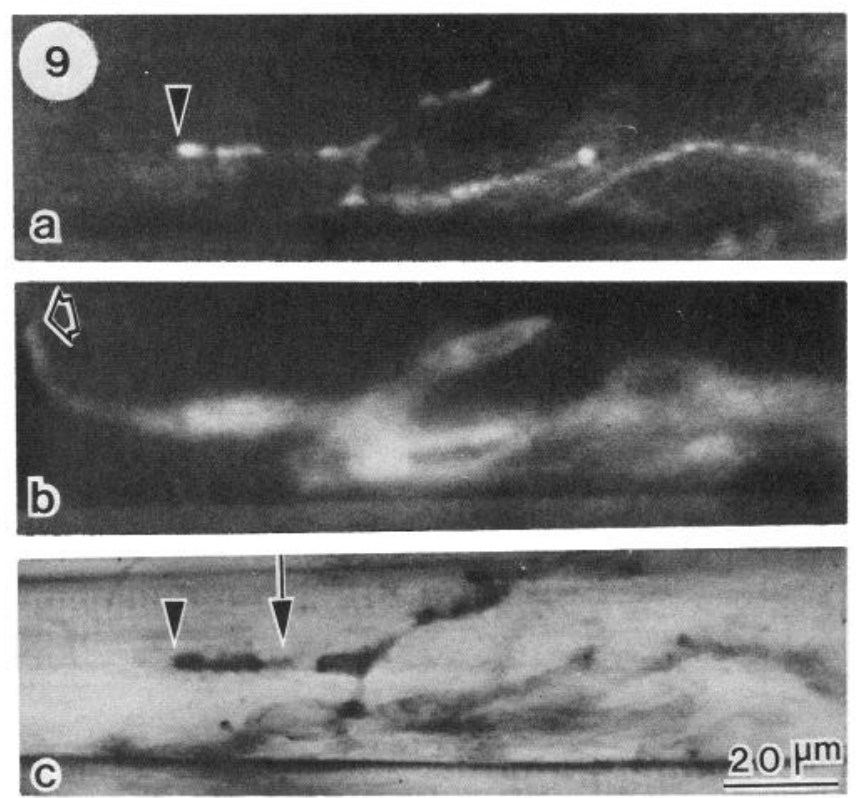

Figure 9. An NMJ stained with the same methods as shown in Figure 8 in a muscle that was not observed previously. The PNA-stained matrix (open arrowhead in $b$ ) is also longer than the 4-Di-2-Asp-stained nerve terminal (solid arrowhead in $a$ ). In contrast to Figure 8 , no cholinesterase staining was seen at the region where PNA staining appears longer than 4-Di-2-Asp staining (c). The arrowhead in $c$ points to the end of the cholinesterase-and nitroblue tetrazolium-stained junctional branch that is correlated with the 4-Di-2-Asp staining of nerve terminals as shown with an arrowhead in $a$. In this junction, the thin nerve terminal cannot be easily differentiated from the dense cholinesterase staining in the black-and-white print, except in the region where cholinesterase staining is absent. The arrow in $c$ indicates a nerve terminal region without accompanying cholinesterase, a sign of sprouting.

in vivo observations. In addition, the dynamic relationship between the motor nerve terminal and the synaptic ECM has been revealed. While precise matching between nerve terminal and synaptic ECM during growth and retraction was commonly seen, mismatching between these two synaptic elements was observed in $16 \%$ of junctional branches viewed repeatedly. This lack of correlation is not caused by the experimental procedure, as similar incongruities also are seen in $15 \%$ of branches when they are examined for the first time. In almost all of these mismatched branches, synaptic matrix is longer than nerve terminal. In addition, control experiments of previously unobserved NMJs, which were located in the same muscles as the identified NMJs or located in the contralateral muscles, showed distributions of total nerve terminal length and total synaptic matrix length similar to those found in the identified NMJs. The results suggest that the synaptic remodeling seen in this study was not caused by the fluorescent staining, illumination, and viewing procedures.

The extensive and widespread remodeling shown in this study is in agreement with and has provided direct evidence to support previous suggestions inferred from studies using histology and electron microscopy of fixed muscles (Wernig et al., 1980; Anzil et al., 1984; Jans et al., 1986). For example, synaptic gutters with cholinesterase reaction product unopposed by nerve terminals have been observed often at the distal tips of NMJs in normal frog muscle. These so-called cholinesterase remnants were interpreted as abandoned parts of the synaptic gutter that 

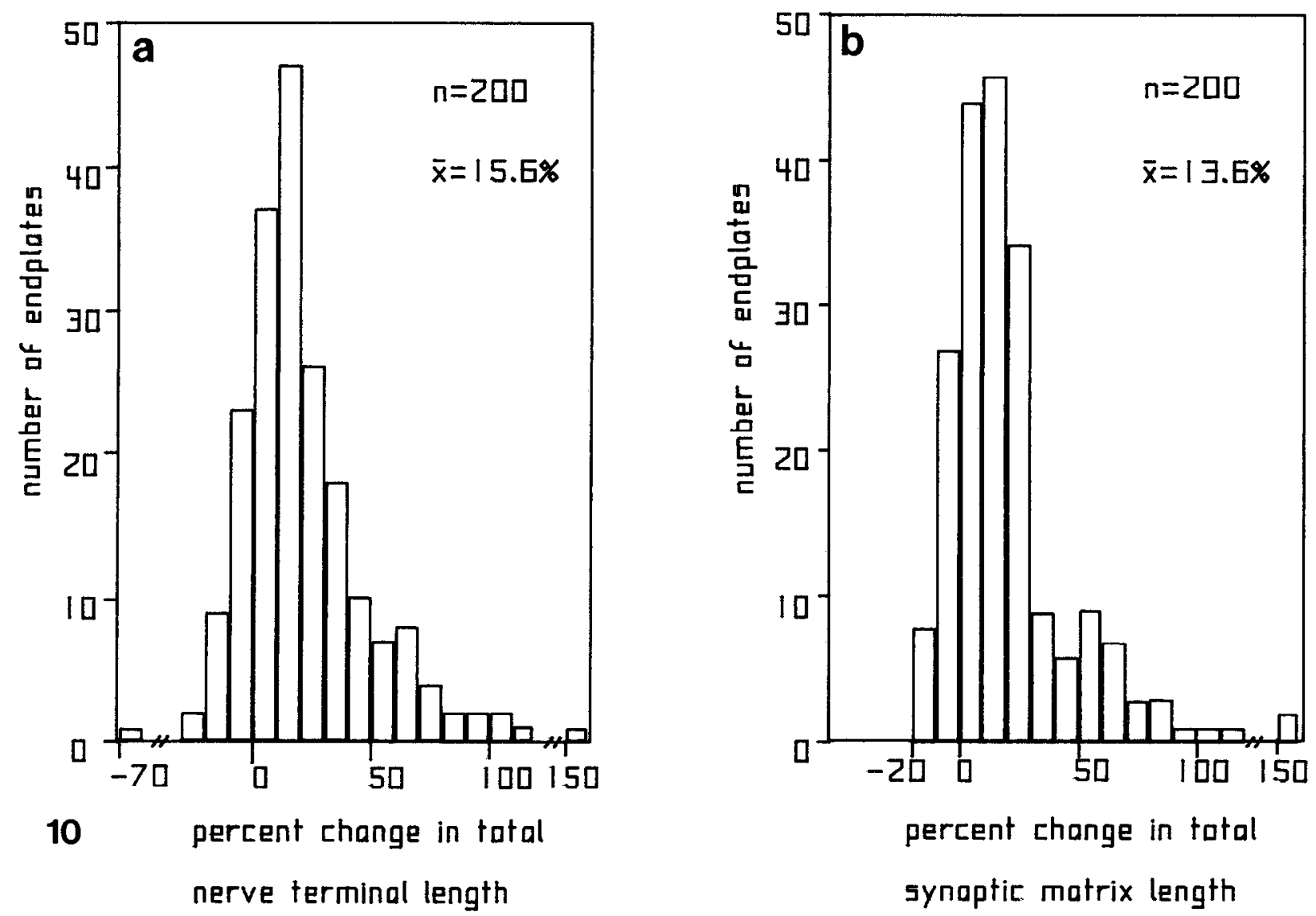

Figure 10. Histograms of percentage change in total nerve terminal length (a) and in total synaptic ECM length (b) for 200 NMJs, observed twice over a period of 2.6-6 months. The average change was $15.6 \%$ growth for the terminal and $13.6 \%$ growth for synaptic ECM.

were previously occupied by a nerve terminal. The present study using repeated observations of the same junctions has revealed the dynamic process of the withdrawal and deletion of junctional branches (also see Herrera et al., 1990). In addition, the present result has indicated that the nerve terminal retracts prior to the synaptic matrix (also see Ko, 1991). With time, the synaptic matrix without overlying terminal gradually diminishes and eventually disappears. The result suggests that the nerve terminal is essential for long-term maintenance of PNA-BMs. In support of this finding, it has been shown that short-term denervation (less than 2 months) of frog NMJs does not affect PNA staining, whereas long-term denervation (2-7.5 months) results in diminution or total loss of PNA binding at NMJs (Somasekhar and Ko, 1991).

The present study has also shown synaptic ECM longer than the nerve terminal at junctions undergoing extension or forming new branches (Figs. 6, 7). There are several possible interpretations of these results. One possibility is that in between observations the nerve tcrminal may have extended, inducing PNABMs at the new site, and then retracted to the starting position, leaving synaptic ECM behind. In this case, one would expect to find cholinesterase remnants because of the retraction of nerve terminals (Wernig et al., 1980) as discussed above at all of the extended PNA-stained branches. However, as shown in Figure 9 , absence of cholinesterase at the region where PNA staining is longer than the nerve terminal has been seen. This result is inconsistent with the above interpretation, unless one also assumes that nerve terminals extend and retract so rapidly that only PNA-BMs, but not cholinesterase, are induced by the growing nerve terminals. This possibility cannot be ruled out with the time resolution provided by the present approach.

Another explanation for the lack of correlation between nerve terminals and synaptic ECM in extending junctional branches is that small filopodia may be present, as seen at living mouse NMJs (Robbins and Polak, 1988), but may not be detectable with 4-Di-2-Asp or other histological staining at the light microscopic level. It is interesting to note that, in the frog NMJs, the extended PNA-stained region is often tens of microns longer than the nerve terminal staining. It is not clear whether all of this region could be occupied by small filopodia. Further study using electron microscopy would help to resolve this question.

After considering the above possibilities, we propose another interpretation: the synaptic matrix may extend in advance of the nerve terminal that would follow later. Anzil et al. (1984) have shown with electron microscopy a Schwann cell process longer than the tip of a thin nerve terminal branch that was thought to be newly formed in an adult NMJ. Since PNA stains predominantly the ECM around Schwann cells at the NMJ (Ko, 1987), the extended PNA staining seen in the present study may be indicative of Schwann cell processes preceding the nerve terminal growth. Schwann cells have been shown to synthesize NGF and other trophic molecules to which growing axons respond (Riopelle et al., 1981; Heumann et al., 1987; VillegasPerez et al., 1988). The question of whether Schwann cells lead axonal outgrowth during development and regeneration has also been raised (Keynes, 1987). Thus, it is possible that during 

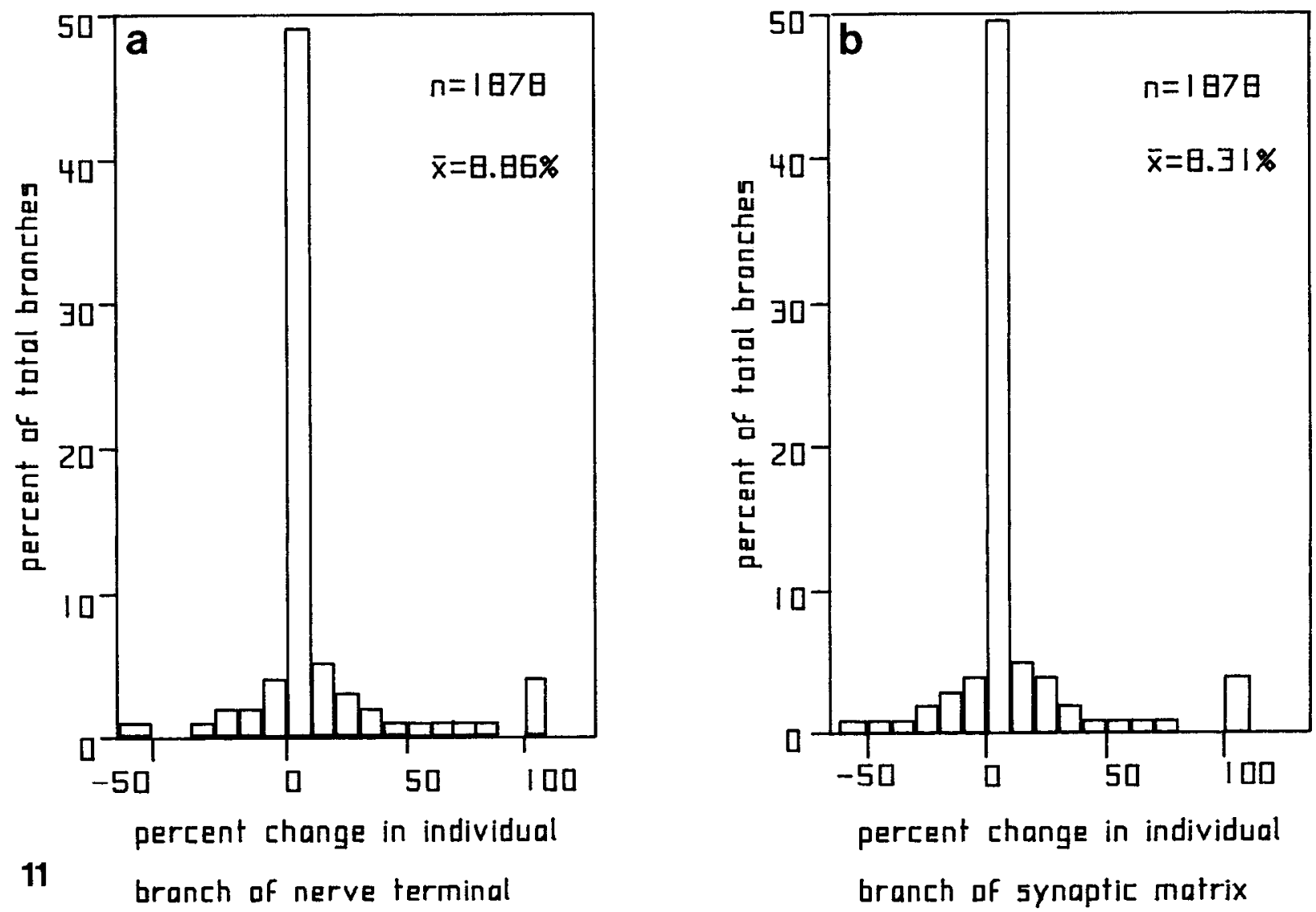

Figure 11. Histograms of percentage change in individual branches of nerve terminals $(a)$ and in individual branch of synaptic ECM $(b)$ for 1878 branches of 200 identified NMJs observed twice over a period of $2.6-6$ months. About $50 \%$ of branches did not show any changes during the interval between observations. The mean change was $8.9 \%$ extension for nerve terminal branch and $8.3 \%$ cxtension for synaptic matrix branch.

synaptic remodeling Schwann cells precede and influence the nerve terminal outgrowth by releasing some kinds of trophic factors or by providing Schwann cell ECM molecules. Pomeroy and Purves (1988) have found changes in the location and the number of glial nuclei associated with each neuron in the parasympathetic ganglion and have suggested that glial cells are active participants in the process of synaptic rearrangement. The present study has shown migration of Schwann cell nuclei along the nerve terminal (also see Herrera et al., 1990). However, it is not known whether this change in the location of Schwann cell nuclei plays any role in synaptic remodeling at the NMJ. Whether the extended distribution of PNA-BMs is indeed indicative of Schwann cell processes preceding nerve terminal outgrowth during synaptic remodeling is also not known. Electron microscopy of identified NMJs showing extensions of PNA staining longer than the nerve terminal following repeated, in vivo observations may provide a way to test this hypothesis. In addition, studies of NMJs that are primarily in active growth, for example, during nerve terminal sprouting, may reveal more junctional branches with extended PNA staining and may provide further support to the hypothesis.

One interesting feature often associated with the growth of NMJs is the presence of a diffuse pattern of synaptic matrix staining in the region of a newly arising terminal. This dispersed distribution of PNA-BMs may be in response to diffusible factors released by forerunning Schwann cells, or it may be asso- ciated with Schwann cell processes that are spreading and exploring new but not yet well-defined territory. The diffuse, PNAstained ECM may gradually become more restricted when Schwann cells are more localized; this may occur even prior to the arrival of nerve terminals. However, without any direct evidence, the above explanation for the diffused PNA staining should be considered merely speculative at this time.

The hypothesis that synaptic ECM precedes nerve terminal outgrowth is compatible with studies of the roles of the ECM in axonal growth and synapse formation. Some ECM components, such as laminin and proteoglycan, have been proven to promote neurite outgrowth (Lander et al., 1982, 1985; Gundersen, 1987; Hantaz-Ambroise et al., 1987). In contrast, s-laminin identified at mammalian NMJs is thought to inhibit neurite extension promoted by laminin (Hunter et al., 1989; Weis et al., 1989). Both adhesion and repulsion may be involved in axonal guidance (Keynes and Cook, 1990). Interestingly, PNA also recognizes a glycoprotein isolated from chick somites, which is capable of causing the collapse of growth cones (Davies et al., 1990). However, it is not known whether the PNA-BM in the frog NMJ is similar to the PNA-binding glycoprotein in the chick somite and is inhibitory to neurite outgrowth. If this were the case, expression of PNA-BMs preceding nerve terminal outgrowth may play an important role by setting an outer boundary that restricts nerve terminals from growing beyond their correct destination. Further studies on biochemical identification and 
characterization of PNA-BMs (Ko et al., 1990) may provide insights into the function of PNA-BMs in synaptic remodeling and maintenance of the NMJ.

\section{References}

Anzil AP, Bieser A, Wernig A (1984) Light and electron microscopic identification of nerve terminal sprouting and retraction in normal adult frog muscle. J Physiol (Lond) 350:393-399.

Balice-Gordon RJ, Lichtman JW (1990) In vivo visualization of the growth of pre- and postsynaptic elements of neuromuscular junctions in the mouse. J Neurosci 10:894-908.

Burden SJ, Sargent PB, McMahan UJ (1979) Acetylcholine receptors in regenerating muscle accumulate at original synaptic sites in the absence of nerve. J Cell Biol 82:412-425.

Chen L, Ko C-P (1988) Dynamic relationship between synaptic extracellular matrix and motor nerve terminal in living frogs. Soc Neurosci Abstr 14:1209.

Davies JA, Cook GMW, Stern CD, Keynes RJ (1990) Isolation from chick somites of a glycoprotein fraction which causes collapses of dorsal root ganglion growth cones. Neuron 4:11-20.

Grinnell AD, Herrera AA (1981) Specificity and plasticity of neuromuscular junctions: long term regulation of motoneuron function. Prog Neurobiol 17:203-282.

Gundersen RW (1987) Response of sensory neurites and growth cones to patterned substrata of laminin and fibronectin in vitro. Dev Biol $121: 423-432$.

Hanta7-Ambroise D, Vigny M, Koenig J (1987) Heparan sulfate proteoglycan and laminin mediate two different types of neurite outgrowth. J Neurosci 7:2293-2304.

Herrera AA, Banner LR (1990) The use and effects of vital fluorescent dyes: observation of motor nerve terminals and satellite cells in living frog muscles. J Neurocytol 19:67-83.

Herrera AA, Werle MJ (1990) Mechanisms of elimination, remodeling, and competition at frog neuromuscular junctions. J Neurobiol 21:73-98.

Herrera AA, Banner LR, Nagaya N (1990) Repeated, in vivo observation of frog neuromuscular junctions: remodeling involves concurrent growth and retraction. J Neurocytol 19:85-99.

Heumann R, Korsching S, Bandtlow C, Thoenen H (1987) Changes of nerve growth factor synthesis in nonneuronal cells in response to sciatic nerve transection. J Cell Biol 104:1623-1631

Hunter D, Shah V, Merlie JP, Sanes JR (1989) A laminin-like adhesive protein concentrated in the synaptic cleft of the neuromuscular junction. Nature 338:229-234.

Inoue S (1986) Video microscopy. New York: Plenum.

Jans H, Salzmann R, Wernig A (1986) Sprouting and nerve retraction in frog neuromuscular junction during ontogenesis and environmental changes. Neuroscience 18:773-781.

Jessell TM, Hynes MA, Dodd J (1990) Carbohydrates and carbohydrate-binding proteins in the nervous system. Annu Rev Neurosci 13:227-255.

Karnovsky MJ (1964) The localization of cholinesterase activity in rat cardiac muscle by electron microscopy. J Cell Biol 23:217-232.

Kelly SS, Anis N, Robbins N (1985) Fluorescent staining of living mouse neuromuscular junctions. Pfluegers Arch 404:97-99.

Keynes R, Cook G (1990) Cell-cell repulsion: clues from the growth cone? Cell 62:609-610.

Keynes RJ (1987) Schwann cells during neural development and regeneration: leaders or followers? Trends Neurosci 10:137-139.

Ko C-P (1987) A lectin, peanut agglutinin, as a probe for the extracellular matrix in living neuromuscular junctions. J Neurocytol 16: $567-576$.

Ko C-P (1991) Peanut agglutinin as a probe for studying plasticity and development of frog neuromuscular junctions. In: Restorative neurobiology and neuroscience (Wernig A, Carmody J, eds), in press. Amsterdam: Elsevier.

Ko C-P, Folsom DB (1990) Induction of synaptic extracellular matrix molecules at ectopic neuromuscular junctions. Dev Brain Res 53: 121-124.

Ko C-P, Najm I, Kraas KM, Baudry M (1990) Identification of peanut agglutinin binding molecules at the frog neuromuscular junction. $\mathrm{J}$ Cell Biol 111:34a.

Lander AD, Fujii DK, Gospodarowicz D, Reichardt LF (1982) Char- acterization of a factor that promotes neurite outgrowth: evidence linking activity to a heparan sulfate proteoglycan. J Cell Biol 94:574585.

Lander AD, Fujii DK, Reichardt LF (1985) Laminin is associated with the "neurite outgrowth-promoting factors" found in condition media. Proc Natl Acad Sci USA 82:2183-2187.

Letinsky MS, DeCino PA (1980) Histological staining of pre- and postsynaptic components of amphibian neuromuscular junctions. J Ncurocytol 9:305-320.

Lichtman JW, Magrassi L, Purves D (1987) Visualization of neuromuscular junction over periods of several months in living mice. $\mathrm{J}$ Neurosci 7:1215-1222.

Lotan R, Skutelsky E, Danon D, Sharon N (1975) The purification, composition, and specificity of the anti-T lectin from peanut (Arachic hypogaea). J Biol Chem 250:8518-8523.

Magrassi L, Purves D, Lichtman JW (1987) Fluorescent probes that stain living nerve terminals. J Neurosci 7:1207-1214.

Nitkin RM, Smith MA, Magill C, Fallon JR, Yao YMM, Wallace BG, McMahan UJ (1987) Identification of agrin, a synaptic organizing protein from Torpedo electric organ. J Cell Biol 105:2471-2478.

Pomeroy SL, Purves D (1988) Neuron/glia relationships observed over intervals of several months in living mice. J Cell Biol 107:11671175.

Purves D, Lichtman JW (1985) Principles of neural development. Sunderland, MA: Sinauer.

Purves D, Voyvodic J (1987) Imaging mammalian nerve cells and their connections over time in living animals. Trends Neurosci 10 398-404.

Purves D, Hadley RD, Voyvodic J (1986) Dynamic changes in the dendritic geometry of individual neurons visualized over periods of up three months in the superior cervical ganglion of living mice. J Neurosci 6:1051-1060.

Purves D, Voyvodic J, Magrassi L, Yawo H (1987) Nerve terminal remodeling visualized in living mice by repeated examination of the same neuron. Science 238:1122-1126.

Riopelle RJ, Boegman RJ, Cameron DA (1981) Peripheral nerve contains heterogeneous growth factors that support sensory neurons in vitro. Neurosci Lett 25:311-316.

Robbins N (1988) Plasticity of motor nerve terminals. In: Nervemuscle cell trophic communication (Fernandez H, Donoso JA, eds), pp 199-215. Boca Raton: CRC.

Robbins N, Polak J (1988) Filopodia, lamellipodia, and retractions at mouse neuromuscular junctions. J Neurocytol 17:545-561.

Sanes JR (1989) Extracellular matrix molecules that influence neural development. Annu Rev Neurosci 12:491-516.

Sanes JR, Cheney JW (1982) Lectin binding reveals a synapse-specific carbohydrate in skeletal muscle. Nature 300:646-647.

Sanes JR, Marshall LM, McMahan UJ (1978) Reinnervation of muscle fiber basal lamina after removal of myofibers. Differentiation of regenerating axons at original synaptic sites. J Cell Biol 78:176-198.

Scotl LJC, Bacou F, Sanes JR (1988) A synapse-specific carbohydrate at the neuromuscular junction: association with both acetylcholinesterase and a glycolipid. J Neurosci 8:932-944.

Somasekhar T, Ko C-P (1991) Effects of denervation on the distribution of peanut agglutinin binding molecules in frog muscles. J Neurocytol 20:65-77.

Villegas-Perez MP, Vidal-Sanz M, Bray GM, Aguayo AJ (1988) Influences of peripheral nerve grafts on the survival and regrowth of axotomized retinal ganglion cells in adult rats. J Neurosci 8:265-280.

Weis J, Hunter DD, Merlie JP, Sanes JR (1989) S-laminin inhibits neurite extension promoted by laminin. Soc Neurosci Abstr 15:164

Wernig $\Lambda$, Herrera $\Lambda \Lambda$ (1986) Sprouting and remodeling at the nervemuscle junction. Prog Neurobiol 27:251-291.

Wernig A, Pecot-Dechavassine M, Stover H (1980) Sprouting and regression of the nerve at the frog neuromuscular junction in norma conditions and after prolonged paralysis with curare. J Neurocytol 9: 277-303.

Wigston DJ (1989) Remodeling of neuromuscular junction in adult mouse soles. J Neurosci 9:639-647.

Wigston DJ (1990) Repeated in vivo visualization of neuromuscular junctions in adult mouse lateral gastrocnemius. J Neurosci 10:17531761 .

Yoshikami D, Okun L (1984) Staining of living presynaptic nerve terminals with selective fluorescent dyes. Nature 310:543-556. 